\title{
Current Trends in Telehealth Applications to Deliver Social Communication Interventions for Young Children with or at Risk for Autism Spectrum Disorder
}

\author{
Jessica Simacek ${ }^{1}$ (D) Marianne Elmquist $^{2} \cdot$ Adele F. Dimian $^{1} \cdot$ Joe Reichle $^{3}$ \\ Accepted: 10 September 2020 / Published online: 12 October 2020 \\ (C) Springer Nature Switzerland AG 2020
}

\begin{abstract}
Purpose of Review Early, intensive, and high-quality interventions can often improve social communication outcomes for children with autism spectrum disorder (ASD). Many children experience barriers to intervention, resulting in significant delays to intervention onset or missed opportunities for intervention altogether. With constant advances in technology, the field is experiencing a rapid increase in investigation of telehealth applications to intervention delivery. This article highlights the current trends in social communication intervention via telehealth used in early intervention practices for children with ASD over the past 5 years, including a brief review of studies (from 2014 to January 2020) and our team's experiences in this area.

Recent Findings Based on our experience and the 22 studies we identified in this area, we describe the current trends in telehealth applications used and how interventions were delivered. We also provide recommendations, limitations, and future directions on this topic.

Summary Telehealth offers innovative intervention delivery options by increasing intervention access, overcoming barriers such as geography and costs of service delivery for young children with ASD.
\end{abstract}

Keywords Telehealth $\cdot$ ASD $\cdot$ Social communication

\section{Introduction}

Early intervention represents a critical factor in improving social communication acquisition for children with ASD [1-3]. Unfortunately, many children encounter barriers to accessing early intervention [4]. These barriers include a shortage of qualified providers of early intervention for this population, geographic areas without adequate service availability, challenges in funding for intervention, and systems that are underprepared to meet the needs of children from

This article is part of the Topical Collection on Communication Disorders

Jessica Simacek

sima0034@umn.edu

1 Institute on Community Integration, University of Minnesota, Minneapolis, MN, USA

2 Department of Educational Psychology, University of Minnesota, Minneapolis, MN, USA

3 Department of Speech, Language, and Hearing Sciences, University of Minnesota, Minneapolis, MN, USA culturally and linguistically diverse populations $[5,6]$ and children from disadvantaged socioeconomic backgrounds [7]. With a growing prevalence in ASD [8], this trend is not likely to reverse without changes in service delivery systems. Since the COVID-19 pandemic, intervention and special education services have been significantly curtailed. Timely solutions are needed to ensure that all children receive access to high-quality early intervention. Telehealth applications represent a potential social communication intervention innovation to address these issues.

The use of telecommunications technology in health care, telehealth, originated and is more prevalent in the medical, psychiatric, and psychological fields. The transition to using telehealth to support intervention activities with children with developmental disabilities emerged somewhat later. Early applications of telehealth in this area include providing intervention and support for students [9], educators [10,11], therapists [12], and parents $[13 \cdot, 14-16]$. Clearly, the evidence of the successful use of telehealth applications in early intervention for children with developmental disabilities is growing. Given current service needs, telehealth applications seem a 
reasonable option to supplement instruction in educational, clinical, and home settings.

The DSM-5® specifies communication and social interaction impairments among key standards yielding an ASD diagnosis [17]. Traditional, in-person intervention services target skills in the social communication domain, including early language skills (e.g., gestures, communication functions (e.g., requests), conversational functions (e.g., conversational repair), vocabulary comprehension, and production) and skills that support socialization during communication (e.g., imitation).

This paper addresses current trends for the use of telehealth to support social communication intervention for young children with ASD. As this paper was in the final stages of development, the world experienced the beginning of the COVID 19 pandemic, introducing an unprecedented urgency for intervention providers to implement telehealth technology at a rapid pace. In this article, we provide considerations and recommendations for providers who are new to implementing telehealth.

We identified 22 studies published between 2014 and January 2020 that met the following inclusion criteria: (a) were experimental or quasi-experimental and published in refereed journals; (b) included children who experienced or were at risk for ASD, with at least one participant under the age of six; (c) reported dependent measures addressing social communication skills; and (d) examined a social communication intervention that included telehealth applications. A PRISMA table of the search process is provided in Appendix 1. The studies were identified by the first three authors across five electronic databases (ERIC via EBSCOhost, LLBA, Academic Search Primer, Web of Science, and PsycINFO), with a literature search that included the following search terms, in 14 search combinations: autism, telehealth, telepractice, teleconsultation, virtual, remote, online verbal language, and social-communication. Twenty-one of the included studies were identified through this search process. One study [18•], was not identified in the search. This study was included based on the aims of the Current Developmental Disorders Reports format, to draw from our team's personal experience and, in this case, to highlight an example of a telehealth-delivered intervention to support social communication skills of children with ASD with more complex communication needs.

\section{Current Trends in Telehealth to Deliver Social Communication Intervention}

We begin by briefly describing telehealth applications including synchronous and asynchronous (i.e., store-and-forward) connections, followed by a brief discussion of the potential benefits of each. We also discuss online learning modules and a learning app. As telehealth includes interaction between an intervention provider and a recipient, standalone modules and apps are often not considered to be telehealth. However, telehealth-delivered interventions are often "packaged" with online modules, which warrants discussion of this modality in this article. Each of the applications discussed can be (and often are) packaged together or combined with in-person intervention delivery.

\section{Synchronous Connection (Live Interaction Among Parties)}

In early intervention for children with ASD, synchronous connection often occurs between an intervention provider, educator, or clinician (herein referred to as "tele-provider") and someone receiving the intervention via telecommunications technology, such as a child or, more often, a caregiver, interventionist, educator, or clinician who is interacting with the child (herein referred to as the "end-user"). In our review, synchronous applications were the most frequently used (12 studies, or $54.5 \%$ of studies used synchronous telehealth alone).

Synchronous telehealth can be a standalone intervention delivery method when families are not receiving in-person intervention with a provider or it can augment a program (e.g., a child receives therapy in a center-based environment and in-home sessions are added to the model via telehealth). For example, Subramaniam et al. provided intervention initially at a clinic with in-person visits from the parents [19॰]. The in-person visits were combined with video-conferencing with parents in their homes, to give the parent time to learn the targeted intervention skills before implementing the strategies with their child in their homes.

A clear advantage to synchronous telehealth is that it allows "live" intervention to be conducted easily in homes, often forgoing travel requirements for both parties. The majority of studies (18 studies, $81.8 \%$ ) from our sample were conducted at least partially in homes. Access to technology and the necessary infrastructure can impose limitations to the advantages of telehealth (e.g., reliable Internet connection); however, successful implementation of telehealth intervention has been demonstrated in rural locations with families with lessthan-optimal Internet connectivity [20•].

Synchronous telehealth can also virtually link clinics or satellite sites (often a community location) with a tele-provider. A potential benefit of this type of connection is to build specialist capacity in an area that typically lacks those resources (e.g., rural location). It can also provide families with a site that has been prepared to receive telehealth connection, which may help avoid some technological barriers [21•]. Teleconnection between sites occurred in a smaller subset of included studies (4 studies; 18\%). Examples of interventions delivered clinic-to-clinic or clinic-to-satellite site included training interventionists in clinics $[22 \bullet, 23 \bullet]$; training parents at local sites including a primary care clinic, a hospital, and a 
school site [21•]; and training early childhood personnel to provide parent training at a local community clinic [24•].

Regardless of location, synchronous connection allows for immediate instructions, feedback, and consultation to be delivered. This can facilitate supervision and fidelity monitoring from a professional (e.g., licensed psychologist) while an interventionist is implementing the intervention directly with a child. This can also enable interdisciplinary service delivery by connecting professionals for wraparound service provision, such as allowing intervention providers working with the child in the home to virtually attend an Individualized Education Plan meeting at the child's school. While these activities can also occur in-person, telehealth may make them more feasible.

Finally, whether telehealth is a reimbursable service modality varies by state, payer, provider type, and the type of service being rendered. Historically, synchronous applications have been more frequently covered than asynchronous applications (Center for Connected Health Policy, https://www. cchpca.org).

\section{Asynchronous Connection (Interaction Among Parties Through Store-and-Forward Technology)}

Asynchronous applications rely on the transmission of audio/ visual or related health information (e.g., imaging) to a teleprovider, who can then review and respond. For example, a tele-provider may ask a parent to record a video of their child communicating during mealtimes and then upload it to a secure online portal. Once uploaded, the tele-provider would view the video to monitor the child's progress and provide feedback to the parent. In our review, asynchronous applications were used less as the only telehealth mode in fewer studies (3 studies, 13.6\%). Studies more frequently included a combination of telehealth approaches (7 studies, 31.8\%).

Benefits to asynchronous applications include that caregivers can often complete intervention sessions and record them during convenient times. It can also provide a window into times of the day when scheduling live intervention sessions is not feasible, such as bedtime. Likewise, tele-providers can collect multiple "video/audio samples" from end-users simultaneously, without scheduling limitations from the provider.

Online Learning Modules and Apps Our review includes online learning modules that were combined with other forms of telehealth. We also describe a study that used an online learning app, Therapy Outcomes By You (TOBY), that contained activities and lessons for parents to implement with their children, as apps like these could be used asynchronously by tele-providers $[25 \bullet]$. Online learning can facilitate the acquisition of introductory information. For example, Neely et al. had interventionists complete an introductory module on naturalistic instruction before further training [23•]. McGarry et al. provided six modules to teach parents how to implement Pivotal Response Training with their children [26•]. In this example, the modules were combined with asynchronous video viewing by teleproviders; however, the authors noted that the asynchronous video viewing was for research purposes only.

Benefits to online learning include that parents/trainees can complete at a convenient time [27•]. This may be essential for families with multiple children, single parents, or other challenges. However, whether parents are likely to complete standalone modules without ongoing contact with a teleprovider is less clear. For example, in the previously mentioned study, McGarry et al. reported a low completion rate for the full sequence of the online learning modules [26•]. In this study, when parents did complete the modules, children's vocalizations, eye contact, and positive affect increased.

\section{Intervention Content and Instructional Components}

Social communication skills targeted in the recent telehealth literature primarily focused on child requests (16 studies; $72.7 \%$ ). Many studies also targeted multiple social communication skills (9 studies; 40.9\%). For example, Guðmundsdóttir et al. demonstrated an increase in social communication skills, including child requests and social attending, language modifiers (e.g., adjectives), and the overall number of words used [20 $0^{\circ}$.

One form of intervention strategy frequently represented in the synchronous telehealth literature has been functional communication training or FCT [16]. Researchers have continued to replicate and refine FCT delivered via telehealth, with this format largely represented (8 studies; $36 \%$, including $[5,18 \bullet$, $28 \bullet, 29 \bullet, 30 \bullet, 31 \bullet, 32 \bullet, 33 \bullet])$. FCT was implemented to increase a communicative alternative to challenging behavior [28 ] and to increase more recognizable communicative alternatives to idiosyncratic responses [18•]. Clearly, FCT appears to be a suitable instructional format for telehealth service delivery for children who are beginning communicators and/or experience challenging behavior.

Naturalistic Developmental Behavioral Intervention (NDBI) models were another instructional format that appeared multiple times in this review (7 studies, 31.8\%). Particularly relevant for younger children, NDBIs combine behavioral principles with developmental and naturalistic approaches (e.g., less structured, more play-based, and child-led approaches to intervention than is commonly associated with behavioral interventions) [34]. For example, Vismara et al. investigated the Parent-Early Start Denver Model, in which parents were provided with synchronous telehealth visits and access to online learning modules, resources, and communication portals via a project website [13•]. Naturalistic and developmental components to this intervention include a focus on relationship development, as well as opportunities for the child to learn being embedded into natural activities, such as sensory-social routines. Other examples of NDBIs delivered via telehealth include Pivotal Response Training [26•], 
RUBI ASD Network PT [24•], and ImPACT Online [35•, $36 \bullet$.

Finally, telehealth can support monitoring maintenance during the process of fading intervention support. Subramaniam et al. provided parents with training in discrete-trial instruction during synchronous telehealth [19•]. Training began with parents attending visits in-person at a clinic; then moved to twice per week with parents in their homes via synchronous telehealth visits; then faded to weekly visits; and finally, faded to biweekly visits. Throughout this gradual fading, parents maintained fidelity.

\section{End-users in Telehealth-Delivered Interventions}

One of the best-documented uses of telehealth applications is training parents to implement intervention strategies to support their child's social communication development (18 studies; $81.8 \%$ ). Parents received training to deliver intervention with their children through various telehealth applications and various parent-coaching procedures. For example, several studies compared self-directed and therapist-assisted interventions delivered via telehealth. Ingersoll et al. compared a selfdirected and a therapist-assisted group using the ImPACT Online training and found that both groups experienced gains in child communication, but only the therapist-assisted group experienced gains in child social skills [35•]. Pickard et al. made a similar comparison and found that both groups reported similar increases in their child's social communication skills [36॰]. However, the parents reported that the therapist assistance was (or would have been) helpful. Applying these findings to recommendations for coaching parents includes that interventionists should consider how to balance the convenience of self-directed online training components, with the importance of opportunities to practice and receive feedback from a tele-provider in a therapist-directed type of format.

Telehealth has also been used to train and provide supervision to interventionists (3 studies; 13.6\%), including a study that involved teaching interventionists on how to coach parents [24•]. Telehealth applications can deliver interventionist training in several ways: (1) to train new interventionists and (2) to support ongoing training. Through telehealth, supervisors can spend more time training and less time traveling.

For example, Neely et al. taught undergraduates to implement incidental teaching strategies with preschool children [22•]. Following online module training for interventionists, the tele-provider viewed asynchronous videos of the trainees implementing the strategies and provided feedback via follow up video-conferencing. Later, Neely et al. provided multitiered provider training that incorporated modules and asynchronous video feedback [23•]. A tele-provider ("specialist") provided training to coaches with an online module; teleproviders (and coaches) viewed recorded videos of the coaches implementing the targeted incidental teaching strategies and provided feedback meetings via videoconferencing between the specialist and the coaches. The program resulted in high levels of fidelity to the teaching strategies and increased requests of the children.

Extending the Reach of Intervention, to Rural Locations and Across the Globe Another significant trend we observed was the extensive reach of telehealth, with many studies actively recruiting children who resided in rural regions and through intercontinental training of interventionists and families. Multiple studies recruited and included families who resided in rural areas $[19 \bullet, 20 \bullet, 21 \bullet, 22 \bullet, 37 \bullet$. For example, RUBI ASD Network Parent Training was delivered through synchronous video-conferencing between a clinic and a satellite site clinic [22•]. Parents located in rural areas came to the site and received coaching via video-conferencing focused on the core and supplemental lessons.

Internationally, multiple studies included families from different countries (highlighted in Fig. 1). Often, families were recruited who resided in rural areas or did not otherwise have access to specialists in early intervention. Barkaia et al. demonstrated successful intercontinental coaching to early intervention providers in Eastern European countries, resulting in improvements in intervention provider implementation and increases in children's requests and vocal imitation [38•]. Guðmundsdóttir et al. demonstrated effective training on a naturalistic intervention sequence over synchronous telehealth to families and a special education teacher in a rural Icelandic location [37॰]. Tsami et al. examined the acceptability and feasibility of FCT with 18 parent-child dyads from multiple countries, recruiting children with parents who did not speak or understand English [28•]. Recommendations for international telehealth-delivered intervention include (a) recruiting of interpreters who were from the same country as the family [28•]; (b) being flexible with using the technology and video-conferencing platform that families have access to and prefer, as this may vary by country [37.]; and (c) anticipating the potential for connectivity problems [37•, 38•].

These accumulated findings demonstrate that telehealth is a tool to innovate intervention delivery for providers to reach children in further areas. It is also a tool for researchers to develop and test interventions that include participant samples from diverse geographic locations, potentially reaching populations of participants whom do not reside near university campus locations.

\section{Recommendations for Beginning Telehealth With Families}

Based on our experience and the available evidence, recommendations for parent-mediated interventions with their young children should be family-centered and routine-based. First, tele-providers can provide assessment for intervention planning and for onboarding a family to telehealth by helping them prepare, troubleshoot, and acclimate to the technology and the intervention. In our previous studies, our protocols 


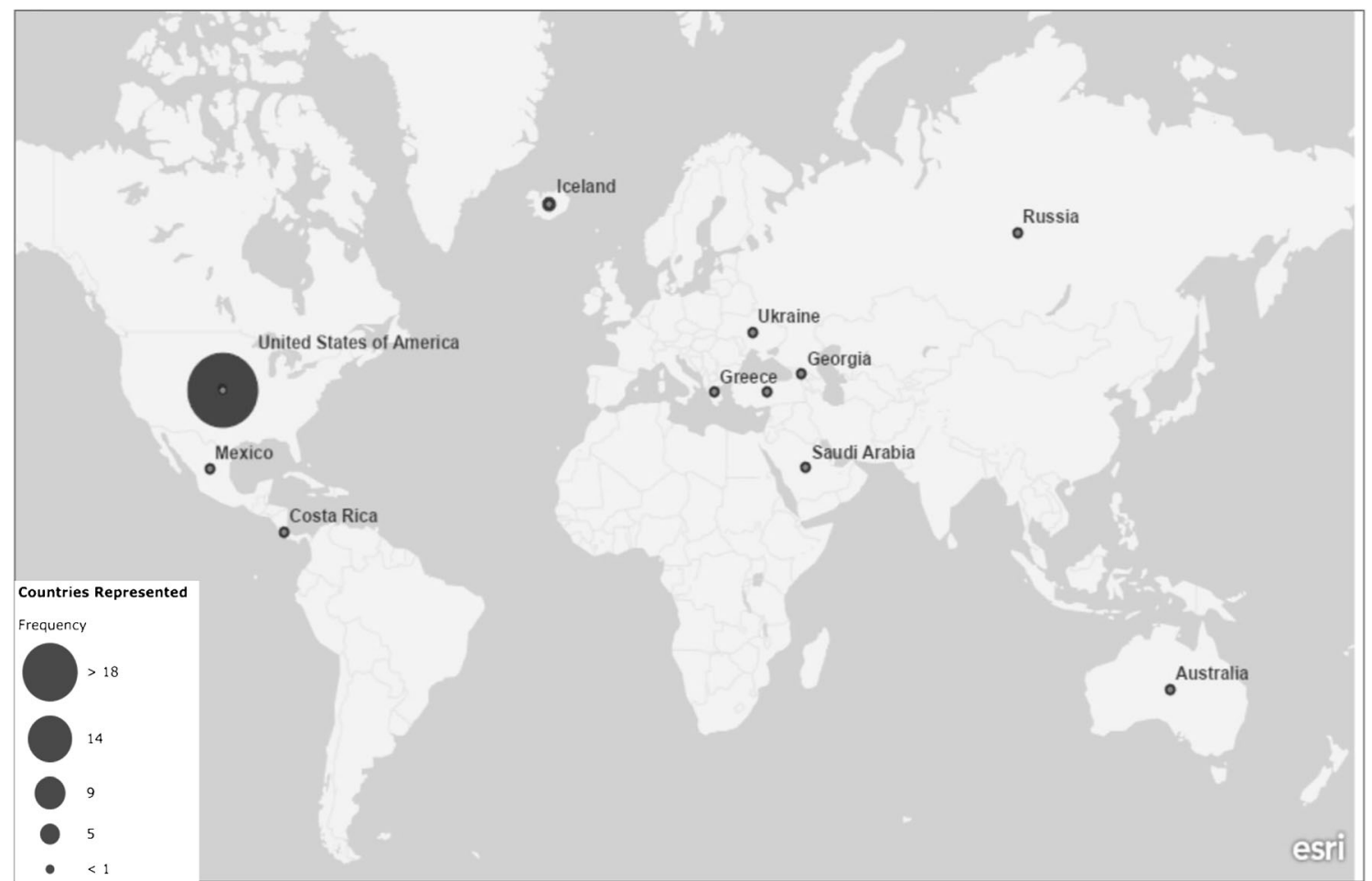

Fig. 1 Global locations of telehealth-supported social communication interventions in the sample of included studies

have begun with a combination of telephone interviews and structured observations [18•, 29•]. Examples of interviews include the Functional Assessment Interview [39], the Inventory of Potential Communicative Acts [40], and the Vineland Adaptive Behavior Scales [41].

In our first sessions with families, we conduct "free play" sessions (approximately 3 to $5 \mathrm{~min}$ of preferred activities available to the child without any demands or restrictions aside from ensuring safety), a strategy we adopted from Wacker et al. [15, 16]. We find that free play helps the child and family adjust to the telehealth and intervention arrangement. Currently, we continue to begin and end most intervention periods with a brief free play session. We also conduct a Structured Descriptive Assessment [42] by implementing several repeated antecedent conditions and routines (e.g., snack time) and measuring the child's potential communicative acts during these times. We compile the assessment information to determine the skills to target and the specific intervention strategies with each child and their parent.

Second, parent education strategies can introduce new concepts to the parent. Parents can receive planning information via email, phone, online modules, or mail, such as providing a list of simple steps that will be implemented or providing instructions on how to prepare for the session (e.g., what items to have available). Another helpful element can be video examples of intervention implementation for parents to view [43•], although this requires video release permission from families.

Third, parents need hands-on experience with implementing strategies with feedback while providing the child with numerous opportunities to respond. While this phase can seemingly occur in different ways, providing some elements of live coaching or closely timed asynchronous feedback can be helpful.

Fourth, the parent should be given opportunities to expand on their use of intervention strategies to support increasingly complex skills for their children [45]. Interventionists should prioritize skills that are important to the family and that promote critical points of social communication development for the child. Some pivotal social communication skills that are foundational for later learning include initiations, imitation, the ability to make requests and choices, naming, responding to requests, and joint attention [46]. As children progress in communication competence, fluency and expansion of communication skills should occur, such as conversational skills.

\section{Conclusions}

\section{Safety, Privacy, and Compliance}

Due to the COVID-19 pandemic, some government agencies have temporarily relaxed certain requirements to allow providers to use telehealth to connect with patients. However, providers should still consider existing requirements and the importance of security of protected health information. For guidance, regulating agencies release recommendations, policies, and resources for adherence to the Health Insurance Portability and Accountability Act [47] and the Family Educational Rights and Privacy Act (FERPA; 20 U.S.C. § 1232g; 34 CFR Part 99) [48], as well as ethical considerations 
on the use of telehealth. Regional telehealth resource centers provide a broad range of telehealth resources (e.g., National Consortium of Telehealth Resource Centers) https://www. telehealthresourcecenter.org)/

\section{Technology Needs}

Many video-conferencing platforms are successfully used for telehealth implementation. Examples from the reviewed studies included GoToMeeting $®$, VSee $®$, and Vidyo®. When selecting a synchronous platform, consider security, HIPAA and/or FERPA, compliance, and features of the platform that enhance usability for the end-user. The use of a virtual private network provides another layer of secure connection [21•].

Capturing high-quality sound during video sessions is another area of consideration. External microphones can enhance the quality of sound, particularly when targeting child communication (e.g., vocalizations) that may be difficult to hear when the child is not close to the computer or telehealth device [20•]. Synchronizing sound and volume during video recordings of telehealth sessions is one area in particular that has been challenging for our team, at times. We have remedied these problems through troubleshooting sound settings outside of intervention sessions with taking test videos of telehealth calls. We then use a dedicated computer with the sound settings set to these tested adjustments and clearly posted instructions not to adjust the sound settings for interventionists who use the computer to run telehealth sessions.

\section{Improving, Not Replacing, In-Person Models}

Despite the clear benefits of providing parent-mediated intervention via telehealth, it is vital that parents alone are not responsible for their children's intervention. Sole reliance on this type of model poses several potential issues: (a) low intervention intensity, (b) a high level of commitment with the potential of additional stress on families, and (c) missed opportunities for the child to receive intervention from professionals with greater levels of expertise and experience and to generalize skills with people outside of their family. Resultantly, many experts caution that telehealth interventions based on parent implementation should be viewed to improve and augment an intervention while in combination with intervention delivered by professionals $[13 \bullet, 23 \bullet]$.

\section{From Feasibility to Effectiveness}

Appropriately for an emerging area of research, the majority of the studies in the current review focused on feasibility, including measures such as fidelity, acceptability, and early efficacy data [43•]. Overall, the majority of studies yielded promising results. This conclusion is consistent with another recent review related to ASD and telehealth [49]. In this previous review, the authors concluded that while results were promising, there was a lack of randomized control trial (RCT) designs [49]. Thus, they indicated a need to move towards studying effectiveness.

In the current review, three studies (13.6\%) used RCTs. Results of these studies were positive, yet mixed. Parsons et al. found an increase in expressive language for a group of children who received the Therapy Outcomes By You app [25•]. Vismara et al. did not find a treatment effect on child social communication skills when comparing a parent-training version of the Early Start Denver Model with a group in a community sample (both groups improved); however, parents' fidelity and ratings of satisfaction improved more in the treatment group [13•]. As previously discussed, Ingersoll et al. found a treatment effect on some, but not all, child measures and an impact on parent measures in a therapist-assisted telehealth intervention compared to a self-directed intervention [35•].

\section{Future Directions}

It is important to consider how telehealth can integrate into established intervention models to address barriers and to optimize outcomes for children and families. The short- and longterm cost-effectiveness of telehealth to deliver social communication intervention for young children with ASD warrants further investigation. There are no clear cost-benefit measures in the studies in our current review. However, previous research indicates a comparable level of efficacy of functional analysis and FCT delivered via telehealth as with other inperson settings, with cost savings reported in the settings that included telehealth and the most substantial cost savings when families were supported in-home via telehealth [14].

Cost analysis needs to be applied to other models of telehealth. For example, the time lag that typically follows after a child is identified as potentially having ASD to when the child receives a diagnosis and then begins intervention is often fraught with delays. This often results in children waiting for long periods of time prior to beginning intervention. In Minnesota, with a cohort of Medicaid-enrolled children, for example, this gap was estimated at 8.99 months of delay [4]. Our research team focuses on investigating parent-mediated intervention via telehealth coaching as a supplement to bridge these delays. If future studies examined the potential cost savings of this type of model, the short-term impact on the cost of the treatment may initially be higher, as more families could access intervention when they historically have not. However, in cost-effectiveness studies of comprehensive (and seemingly expensive) early intervention models, the long-term cost savings far offset the costs of the interventions themselves [50,51]. Piccininni et al. estimated that if wait times to accessing early intervention were eliminated in Ontario, Canada, the lifetime savings would be $\$ 267,000$ per individual [50]. Supplemental models of telehealth to enhance and improve early intervention should be a continued area of future research and should include cost-benefit comparisons that take into account the potential for long-term cost savings. 
There is a similar potential for expansion of telehealth into early childhood education. To embed telehealth into this model potentially allows for home visits to occur more frequently or for expanding center-based programs to include a home connection component. These examples could increase the dosage of the interventions, allowing educators to see children multiple times per week and to serve children across multiple settings. Meadan et al. demonstrated feasibility of coaching an early childhood specialist based in an early childhood center to coach families in their homes [43•]. However, this model would need to be tested at a larger scale to inform its impact on feasibility and effectiveness for children, families, and educators.

\section{Future Telehealth Technologies}

Researchers are incorporating new technological developments into novel intervention delivery approaches. When reviewing the recent literature, we screened a large number of studies that included keywords based on virtual reality. These studies did not meet our inclusion criteria due to a missing interaction between a tele-provider and a tele-user or including participants in the wrong age range. However, examples in the literature are beginning to emerge, such as the use of virtual reality in facial affect recognition of adolescents with ASD [52].

There are two other telehealth applications that did not emerge in our search of the literature: (a) remote monitoring, in which technology allows a tele-provider to monitor the tele-user via technology (e.g., wearable technology to collect heart rate data [44], and (b) mHealth, the use of mobile technology to share health and public health information (e.g., text alerts to notify tele-users with a chronic condition to refill a medication). There may be particular relevance for future exploration of remote monitoring to monitor speech samples for children with ASD or other home-based measures. We anticipate that over the next 5 years, there will be a greater focus in these areas.

\section{Compliance with Ethical Standards}

Conflict of Interest The authors declare that they have no conflicts of interest.

Human and Animal Rights and Informed Consent This article does not contain any studies with human or animal subjects performed by any of the authors.

\section{Appendix}

Fig. 2 PRISMA flow diagram of citations reviewed. Please note that we opted to include an additional article from our team $[18 \bullet]$ that was not located in the initial search but does meet the inclusion criteria. We used examples from this work to report experience in interventions delivered via telehealth from our team. We also included a study from January, 2020 (Suess et al., 2020)

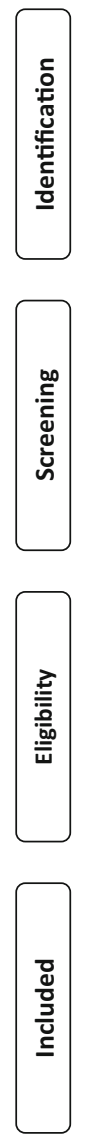

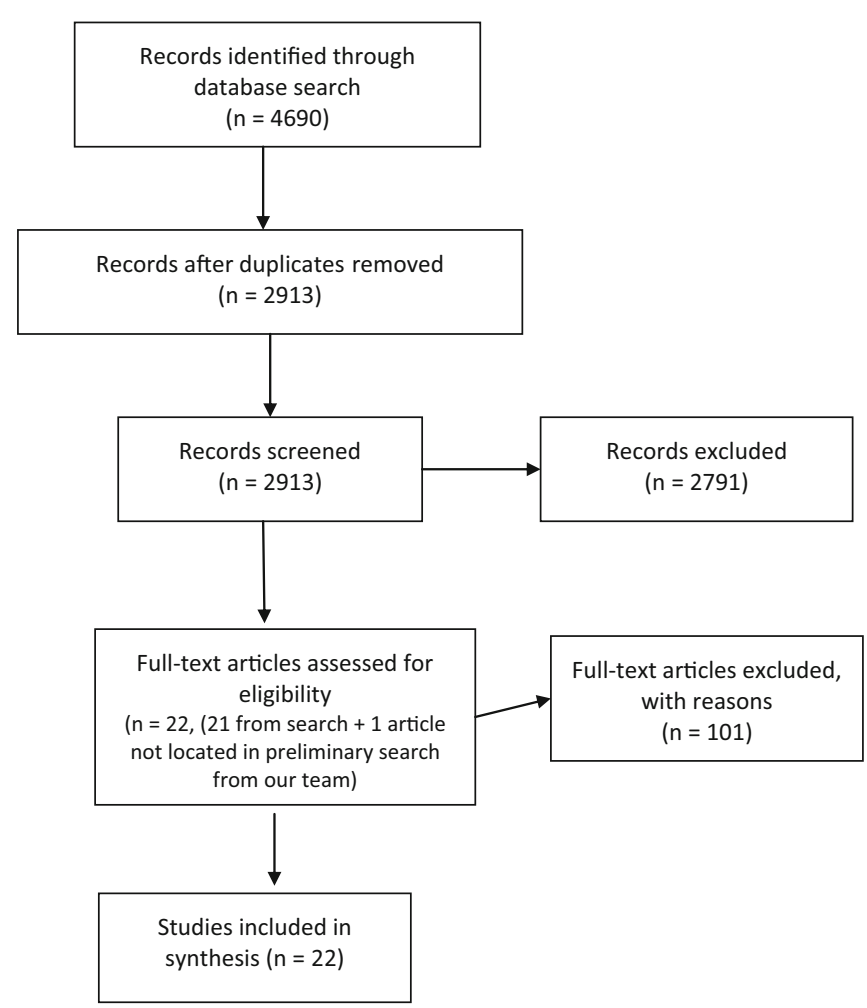




\section{References}

Papers of particular interest, published recently, have been highlighted as:

- Of importance

1. Hyman SL, Levy SE, Myers SM. Council on Children with Disabilities, Section on Developmental and Behavioral Pediatrics. Identification, evaluation, and management of children with autism spectrum disorder. Pediatrics. 2020;145:1-64.

2. Eldevik S, Hastings RP, Hughes JC, Jahr E, Eikeseth S, Cross S. Using participant data to extend the evidence base for intensive behavioral intervention for children with ASD. Am J Intellect Dev Disabil. 2010;115:381-405.

3. Virués-Ortega J. Applied behavior analytic intervention for ASD in early childhood: meta-analysis, meta-regression and dose-response meta-analysis of multiple outcomes. Clin Psychol Rev. 2010;30: 387-99.

4. Dimian AF, Symons FJ, Wolff JJ. Delay to early intensive behavioral intervention and educational outcomes for a Medicaidenrolled cohort of children with autism. J Autism Dev Disord. 2020:1-13. https://doi.org/10.1007/s10803-020-04586-1.

5. Liptak GS, Benzoni LB, Mruzek DW, Nolan KW, Thingvoll MA, Wade CM, et al. Disparities in diagnosis and access to health services for children with autism: data from the National Survey of Children's Health. J Dev Behav Pediatr. 2008;29:152-60.

6. Magaña S, Lopez K, Aguinaga A, Morton H. Access to diagnosis and treatment services among Latino children with autism spectrum disorders. Intellect Dev Disabil. 2013;51:141-53.

7. Nguyen CT, Krakowiak P, Hansen R, Hertz-Picciotto I, Angkustsiri K. Sociodemographic disparities in intervention service utilization in families of children with autism spectrum disorder. J Autism Dev Disord. 2016;46:3729-38.

8. Baio J, Wiggins L, Christensen DL, Maenner MJ, Daniels J, Warren Z, et al. Prevalence of ASD spectrum disorder among children aged 8 years-ASD and developmental disabilities monitoring network, 11 sites, United States, 2014. MMWR Surveill Summ. 2018;67:1-23.

9. Machalicek W, O'Reilly MF, Rispoli M, Davis T, Lang R, Franco $\mathrm{JH}$, et al. Training teachers to assess the challenging behaviors of students with ASD using video tele-conferencing. Educ Trainin Autism Dev Disabil. 2010;45:203-15.

10. Barretto A, Wacker DP, Harding J, Lee J, Berg WK. Using telemedicine to conduct behavioral assessments. J Appl Behav Anal. 2006;39:333-40.

11. Gibson JL, Pennington RC, Stenhoff DM, Hopper JS. Using desktop videoconferencing to deliver interventions to a preschool student with autism. Top Early Child Spec Educ. 2010;29:214-25.

12. Vismara LA, Young GS, Stahmer AC, Griffith EM, Rogers SJ. Dissemination of evidence-based practice: can we train therapists from a distance? J Autism Dev Disord. 2009;39:1636-51.

13. Vismara LA, McCormick CE, Wagner AL, Monlux K, Nadhan A, Young GS. Telehealth parent training in the Early Start Denver Model: results from a randomized controlled study. Focus on ASD and Other Developmental Disabilities. 2018;33:67-79 This study compared to the effects of parent training on the Early Start Denver Model delivered via telehealth with a group receiving community intervention. The study used both synchronous and asynchronous modalities to coach parents to increase child spontaneous utterances, imitative functional play, and joint attention outcomes.

14. Lindgren S, Wacker D, Suess A, Schieltz K, Pelzel K, Kopelman T, et al. Telehealth and autism: Treating challenging behavior at lower cost. Pediatrics. 2016;137(Supplement 2):S167-75.
15. Wacker DP, Lee JF, Dalmau YC, Kopelman TG, Lindgren SD, Kuhle J, et al. Conducting functional analyses of problem behavior via telehealth. J Appl Behav Anal. 2013;46:31-46.

16. Wacker DP, Lee JF, Dalmau YC, Kopelman TG, Lindgren SD, Kuhle J, et al. Conducting functional communication training via telehealth to reduce the problem behavior of young children with autisms. J Dev Phys Disabil. 2013;25:35-48.

17. American Psychiatric Association. Diagnostic and statistical manual of mental disorders (DSM-5®). American Psychiatric Pub; 2013.

18. Dimian AF, Elmquist M, Reichle J, 31J. Teaching communicative responses with a speech-generating device via telehealth coaching. Advances in Neurodevelopmental Disorders. 2018; 1; 2:86-99. This study-utilized telehealth to train parents to implement communication interventions with speech-generating devices.

19. Subramaniam S, Brunson LY, Cook JE, Larson NA, Poe SG, Peter CC. Maintenance of parent-implemented discrete-trial instruction during videoconferencing. J Behav Educ. 2017;26:1-26 This study evaluated the effects of training parents in discrete trial training through a combination of in-person and synchronous telehealth modalities on maintenance of the parent implementation and targeted child skills.

20. Guðmundsdóttir K, Ala'i-Rosales S, Sigurðardóttir ZG. Extending caregiver training via telecommunication for rural Icelandic children with ASD. Rural Spec Educ Q. 2019;38:26-42 This study used telehealth to deliver a parent-training program for families living in rural areas with low-speed internet access. Parents were coached with synchronous methods to increase child social attending and requesting.

21. Bearss K, Burrell TL, Challa SA, Postorino V, Gillespie SE, Crooks C, et al. Feasibility of parent training via telehealth for children with ASD spectrum disorder and disruptive behavior: a demonstration pilot. J Autism Dev Disord. 2018;48:1020-30 This study evaluated the feasibility of delivering RUBI-PT via telehealth. Parents were coached synchronously.

22. Neely L, Rispoli M, Boles M, Morin K, Gregori E, Ninci J, et al. Interventionist acquisition of incidental teaching using pyramidal training via telehealth. Behav Modif. 2019;43:711-33 This study used telehealth to implement a pyramidal training program to train clinicians to implement incidental teaching strategies to increase child requesting.

23. Neely L, Rispoli M, Gerow S, Hong ER. Preparing interventionists via telepractice in incidental teaching for children with autism. J Behav Educ. 2016;25:393-416 This study used telehealth to train clinicians in implementing incidental teaching targeted to child requests. Both synchronous and asynchronous telehealth applications were used.

24. Hoffmann AN, Bogoev BK, Sellers TP. Using telehealth and expert coaching to support early childhood special education parentimplemented assessment and intervention procedures. Rural Spec Educ Q. 2019;38:95-106 This study used telehealth to train Early Childhood Special Education behavior specialists to support parent-implemented behavioral procedures and interventions.

25. Parsons D, Cordier R, Lee H, Falkmer T, Vaz S. A randomised controlled trial of an information communication technology delivered intervention for children with autism spectrum disorder living in regional Australia. J Autism Dev Disord. 2019;49:569-81 This study evaluated the effectiveness of a tablet-based early intervention application developed to supplement intervention.

26. McGarry E, Vernon T, Baktha A. Brief report: a pilot online pivotal response treatment training program for parents of toddlers with autism spectrum disorder. J Autism Dev Disord. 2019;12:1-8 This study evaluated the use of a self-directed online program to train parents on pivotal response treatment. 
27. Douglas SN, Kammes R, Nordquist E. Online communication training for parents of children with autism spectrum disorder. Commun Disord Q. 2018;39:415-25 This study used telehealth to train parents to implement POWR to increase child communication. Parents were trained using an asynchronous telemodel

28. Tsami L, Lerman D, Toper-Korkmaz O. Effectiveness and acceptability of parent training via telehealth among families around the world. J Appl Behav Anal. 2019;52:1113-29 This international study investigated a training model of telehealth to coach parents on functional analysis and FCT across multiple countries to decrease challenging behavior and to increase child requests, as well as treatment acceptability.

29. Simacek J, Dimian AF, McComas JJ. Communication intervention for young children with severe neurodevelopmental disabilities via telehealth. J Autism Dev Disord. 2017:47:744-67 This study examined the effects of parent-implemented functional communication training delivered via synchronous telehealth coaching on child requests during routines.

30. Benson SS, Dimian AF, Elmquist M, Simacek J, McComas JJ, Symons FJ. Coaching parents to assess and treat self-injurious behaviour via telehealth. J Intellect Disabil Res. 2018;62:1114-23 This study coached parents synchronously via telehealth to implement assessment and intervention (functional communication training) procedures to reduce self-injurious behaviors and to increase child requests.

31. Suess AN, Romani PW, Wacker DP, Dyson SM, Kuhle JL, Lee JF, et al. Evaluating the treatment fidelity of parents who conduct inhome functional communication training with coaching via telehealth. J Behav Educ. 2014;23:34-59 This study examined parent's implementation fidelity of functional communication training conducted with telehealth

32. Suess AN, Schieltz KM, Wacker DP, Detrick J, Podlesnik CA. An evaluation of resurgence following functional communication training conducted in alternative antecedent contexts via telehealth. J Exp Anal Behav. 2020;113:278-301 This study examined the use of parent-implemented functional analyses and functional communication training with synchronous coaching via telehealth.

33. Tsami L, Lerman DC. Transfer of treatment effects from combined to isolated conditions during functional communication training for multiply controlled problem behavior. J Appl Behav Anal. 2020;53:649-64 This study examined the use of telehealth to coach parents synchronously to implement functional analyses and FCT to decrease challenging behavior and to increase child requests.

34. Schreibman L, Dawson G, Stahmer AC, Landa R, Rogers SJ, McGee GG, et al. Naturalistic developmental behavioral interventions: empirically validated treatments for autism spectrum disorder. J Autism Dev Disord. 2015;45:2411-28.

35. Ingersoll B, Wainer AL, Berger NI, Pickard KE, Bonter N. Comparison of a self-directed and therapist-assisted telehealth parent-mediated intervention for children with ASD: a pilot RCT. J Autism Dev Disord. 2016;46:2275-84 This RCT study compared a self-directed and a therapist-assisted implementation of ImPACT Online.

36. Pickard KE, Wainer AL, Bailey KM, Ingersoll BR. A mixedmethod evaluation of the feasibility and acceptability of a telehealth-based parent-mediated intervention for children with autism spectrum disorder. Autism. 2016:845-55 This study evaluated ImPACT Online intervention training for parents through either a self-directed or a therapist-assisted program.
37. Guðmundsdóttir K, Sigurðardóttir ZG, Ala’i-Rosales S. Evaluation of caregiver training via telecommunication for rural Icelandic children with ASD. Behav Dev Bull. 2017;22:215 This international study examined the use of telehealth to train parents on implementation of a sequence of naturalistic steps for child communication initiations and responses.

38. Barkaia A, Stokes TF, Mikiashvili T. Intercontinental telehealth coaching of therapists to improve verbalizations by children with autism. J Appl Behav Anal. 2017;50:582-9 This study investigated a therapist-coaching model across countries to target child verbalizations.

39. O'Neill RE, Horner RH, Albin RW, Storey K, Sprague JR. Functional analysis of problem behavior: a practical assessment guide: Sycamore Publishing Company; 1990.

40. Sigafoos J, Woodyatt G, Keen D, Tait K, Tucker M, RobertsPennell $\mathrm{D}$, et al. Identifying potential communicative acts in children with developmental and physical disabilities. Commun Disord Q. 2000;21:77-86.

41. Sparrow SS, Cicchetti DV, Saulnier CA. Vineland Adaptive Behavior Scales, (Vineland-3). Antonio: Psychological Corporation; 2016.

42. Anderson CM, Long ES. Use of a structured descriptive assessment methodology to identify variables affecting problem behavior. J Appl Behav Anal. 2002;35:137-54.

43. Meadan H, Snodgrass MR, Meyer LE, Fisher KW, Chung MY, Halle JW. Internet-based parent-implemented intervention for young children with autism: a pilot study. J Early Interv. 2016;38: 3-23 This study examined the effects of parent-implemented naturalistic intervention delivered with telehealth coaching on their child's communication skills.

44. Ingersoll B, Dvortcsak A. Teaching social communication to children with autism: a practitioner's guide to parent training and a manual for parents. New York: NY. Guilford Press; 2009.

45. Koegel LK, Koegel RL, Shoshan Y, McNerney E. Pivotal response intervention II: preliminary long-term outcome data. J Assoc Pers Sev Handicaps. 1999;24:186-98.

46. Act A. Health insurance portability and accountability act of 1996 Public law. 1996;104:191.

47. Fry BG, Therese M, Weckmueller BL. The family educational rights and privacy act of 1974. Student Records Management: A Handbook. 1997;43.

48. Knutsen J, Wolfe A, Burke BL, Hepburn S, Lindgren S, Coury D. A systematic review of telemedicine in autism spectrum disorders. Rev J Autism Dev Disord. 2016;3:330-44.

49. Piccininni C, Bisnaire L, Penner M. Cost-effectiveness of wait time reduction for intensive behavioral intervention services in Ontario. Canada JAMA Pediatr. 2017;171:23-30.

50. Bekele E, Crittendon J, Zheng Z, Swanson A, Weitlauf A, Warren $Z$, et al. Assessing the utility of a virtual environment for enhancing facial affect recognition in adolescents with ASD. J ASD Dev Disord. 2014;44:1641-50.

51. Chasson GS, Harris GE, Neely WJ. Cost comparison of early intensive behavioral intervention and special education for children with ASD. J Child Fam Stud. 2007;16:401-13.

52. Malasinghe LP, Ramzan N, Dahal K. Remote patient monitoring a comprehensive study. J Ambient Intell Humaniz Comput. 2019;10: $57-76$.

Publisher's Note Springer Nature remains neutral with regard to jurisdictional claims in published maps and institutional affiliations. 